\title{
Nuove tecnologie per la rimozione dei prodotti farmaceutici nelle acque reflue
}

\author{
Rosa Urli ${ }^{1}$
}

${ }^{1}$ Affiliation not available

\begin{abstract}
Negli ultimi anni si ha la crescente preoccupazione per la presenza e il destino di farmaci nell'ambiente acquatico, i cui potenziali effetti ambientali sono in gran parte sconosciuti quando tali composti sono compresenti in miscela. La volontà di impedire completamente il loro ingresso negli ecosistemi, mossa dalla precauzione, stimola la ricerca su metodi di trattamento alternativi, tra questi il trattamento combinato con ozono e ultrasuoni. Da svariati studi si evince che le rese del processo per la rimozione di singoli composti farmaceutici selezionati sono efficaci ma nella realtà gli impianti devono fronteggiare reflui dalle caratteristiche sempre diverse e con miscele di contaminanti in differenti concentrazioni. Bisogna dunque mirare alla caratterizzazione di tali prodotti, comprendere meglio i processi e migliorare le tecnologie in modo da poter perseguire l'obiettivo.
\end{abstract}

\section{I contaminanti emergenti e i prodotti farmaceutici}

I contaminanti emergenti rappresentano una categoria di inquinanti, definiti persistenti, che mostrano una forte resistenza alla decomposizione, soprattutto se affrontati con i metodi tradizionali di trattamento delle acque reflue. L'urgente necessità di aggiornare e ottimizzare i processi di ri- 
mozione nasce dalla valutazione del rischio che la loro presenza nelle acque comporta, soprattutto in termini di rischio ecologico ${ }^{1}$. Infatti, anche in concentrazioni minime (al di sotto dei $\mu \mathrm{g} / \mathrm{L}$ ), il loro potenziale tossico e inibitorio genera danni agli ecosistemi acquatici ${ }^{23}$ e di conseguenza all'uomo.

Tali contaminanti derivano principalmente da prodotti per la cura personale, ormoni, fertilizzanti e industrie farmaceutiche ${ }^{2}$. Nello specifico dei prodotti farmaceutici, quelli più frequentemente rilevati sono il diclofenac (DCF), un agente antinfiammatorio, il sulfametossazolo (SMX) e l'amoxicillina (AMX), due antibiotici, e la carbamezepina (CBZ), un farmaco anticonvulsivante e stabilizzante dell'umore ${ }^{3456}$. Gli effetti delle appena citate sostanze riversate nell'ambiente si ripercuotono soprattutto e direttamente sulla fauna acquatica.

\section{Soluzioni proposte}

La letteratura scientifica offre svariate proposte con il fine di aumentare l'efficienza di rimozione dei prodotti farmaceutici dalle acque reflue, tra cui adsorbimento, tecnologie a membrana, processi di ossidazione avanzata e altre soluzioni alternative ${ }^{1}$, come l'elettrocoagulazione ${ }^{78}$, la cui scelta di utilizzo dovrà sicuramente essere coadiuvata da criteri decisionali obiettivi e analitici, soprattutto dalla prospettiva della sostenibilità ${ }^{9}$.

L'ozono $\left(\mathrm{O}_{3}\right)$ ha dimostrato di essere un ossidante efficiente per la rimozione di vari inquinanti organici. L'ozonizzazione prevede la decomposizione di $\mathrm{O}_{3}$ in acqua che forma radicali idrossilici $(\cdot \mathrm{OH})$ i quali reagiscono con i composti organici disciolti ${ }^{10}$. Purtroppo, in virtù della natura degli inquinanti, possono aversi potenziali rischi ambientali a causa della formazione di sottoprodotti che comportano un incremento della tossicità, indipendentemente dalla dose di $\mathrm{O}_{3}$ utilizzata ${ }^{111213}$. Un'altra tecnologia fortemente in crescita, in diversi campi di applicazione, è la Sonolisi, ovvero 
la produzione di energia con ultrasuoni (US), un processo versatile in grado di eliminare in modo efficiente una varietà di inquinanti e agenti patogeni ${ }^{14131516}$ e facilmente integrabile con altre tecnologie. Gli US, infatti, possono essere impiegati in diverse fasi del processo di trattamento delle acque reflue, nella linea di trattamento fanghi, nella solubilizzazione dei fanghi attivi dei rifiuti e altro ${ }^{17181920}$. Test hanno dimostrato che l'aumento della densità degli US o il tempo di irradiazione ultrasonora influenza la quantità di sostanze solubilizzate ${ }^{18}$, anche nel caso della conversione dei composti farmaceutici, migliorata anch'essa all'aumentare della potenza applicata, alle condizioni acide e in presenza di aria disciolta ${ }^{5}$. L'efficienza del singolo processo, inoltre, è incrementabile tramite l'utilizzo di catalizzatori, tra i più comuni il biossido di titanio ${ }^{4}$. Tra gli svantaggi sono da annoverarsi il consumo di energia e l'incremento di torbidità dell'acqua ${ }^{13}$.

Da alcuni risultati si evince come la sonolisi sia più efficiente dell'ozonizzazione ${ }^{21} 22$ ma anche che, se applicati congiuntamente (Fig. 1), portano ad effetti sinergici aumentando le efficienze di rimozione, motivo per il quale diversi sono gli studi che sperimentano tale metodo "ibrido", anche insieme ad ulteriori trattamenti ${ }^{31721919}$.

\section{L'ozonizzazione potenziata con ultrasuoni}

Come esempio illustrativo, si riporta lo studio effettuato da ${ }^{3}$. L'obiettivo dello studio era quello di valutare le rese di rimozione e le velocità di degradazione di composti farmaceutici sia nel caso di singole soluzioni sia nel caso di miscele complesse, una volta considerando come trattamento la sola ozonizzazione e successivamente combinando il processo di ozonizzazione con gli ultrasuoni $\left(\mathrm{O}_{3} / \mathrm{US}\right)$.

Ipotizzando che le velocità delle reazioni non dipendano dalle concentrazioni dei componenti, ovvero che un reagente si consumi sempre alla stessa velocità fino alla sua completa scomparsa ${ }^{53}$, sono state utilizzate concentrazioni di contaminanti $(10 \mathrm{mg} / \mathrm{L})$ superiori a quelle che solitamente si 
riscontrano nella realtà. L'ozonizzazione era garantita da un sistema dotato di lampade ultraviolette che consentivano di separare le molecole di ossigeno presenti in un flusso d'aria costante per la produzione dell'ozono. L'ozono necessario, tramite un diffusore, veniva poi spinto in un reattore, al centro del quale era posizionata una sonda. Un generatore collegato ad essa consentiva di produrre ultrasuoni e quindi di immettere gli stessi nella miscela contenuta nel reattore, combinando di fatto i due processi.

Per la sola ozonizzazione, le rimozioni di DCF, SMX e CBZ sono state rispettivamente $73 \%, 51 \%$ e $59 \%$ in singole soluzioni e $73 \%, 49 \%$ e $55 \%$ in miscela, ottenute in 40 min e con un flusso di $\mathrm{O}_{3}$ di 3,3 g/h. Tali percentuali sono simili e fanno intendere che le velocità delle reazioni e la quantità di ozono necessaria sarebbero diverse se si volesse ottenere una rimozione totale dei singoli prodotti farmaceutici. Questo è dovuto probabilmente alle caratteristiche di reattività dei componenti, alla loro struttura molecolare e alla competitività che possono mostrare quando sono compresenti. Infatti, i valori diminuiscano leggermente nel caso della miscela rispetto alle singole soluzioni poiché l'ozono non è selettivo e reagisce con tutti i composti contemporaneamente ${ }^{23}$.

In un secondo momento, introducendo gli US in modo costante, è stato fatto variare il flusso di $\mathrm{O}_{3}$. Si è visto come, anche per valori inferiori a $3,3 \mathrm{~g} / \mathrm{h}$ di $\mathrm{O}_{3}$, si ha subito un netto potenziamento del processo. Ad esempio, considerando le stesse condizioni di 3,3 $\mathrm{g} / \mathrm{h}$ di $\mathrm{O}_{3}$ per $40 \mathrm{~min}$, il DCL è stato rimosso al 94\% e il SMX al 61\% in singole soluzioni, un po' meno in miscela. Questo grazie alla maggiore produzione di radicali idrossilici, facilitata dalla rottura, da parte degli US, delle molecole di ozono in microbolle che possono maggiormente reagire con la fase liquida e allo stesso tempo intensificano l'effetto di cavitazione ${ }^{3}$. 


\section{Conclusioni}

La presente trattazione vuole mettere in evidenza un'attuale problematica, quale la presenza di prodotti farmaceutici nell' ambiente, e quelle che sono le soluzioni proposte dalla letteratura scientifica, in particolare il trattamento ibrido $\mathrm{O}_{3} / \mathrm{US}$. In generale, si può affermare che gli effetti dell'ozonizzazione sono amplificati dalla sonolisi e che è possibile ridurre la dose chimica di reagente. D'altra parte, non può essere definita una dose ottimale che consenta contemporaneamente di eliminare tutti i composti bersaglio perché il potenziale di rimozione dei contaminati dipende dagli stessi.

Un'altra importante conclusione è che la presenza simultanea di DFC, SMX e CBZ non costituisce un impedimento nel lasciarsi degradare, in questo senso sono poco competitivi, e dunque che l'uso di ozono/ultrasuoni può essere applicato alle reali acque reflue come ulteriore trattamento biologico poiché, dalle sperimentazioni, sono state dimostrate valide rese di rimozione.

Le variabili di cui tener conto sono tante, per questo si ha la necessità di incrementare la ricerca su questa valida soluzione tecnologica, in modo da acquisire tutte le competenze per poter rimuovere i prodotti farmaceutici nell'ambiente.

\section{References}

1.Lin, X. et al.. Occurrence and risk assessment of emerging contaminants in a water reclamation and ecological reuse project. Science of The Total Environment 744, 140977 (2020).

2.Rathi, B. S., Kumar, P. S. \& Show, P.-L. A review on effective removal of emerging contaminants from aquatic systems: Current trends and scope for further research. Journal of Hazardous 
Materials 124413 (2020) doi:10.1016/j.jhazmat.2020.124413.

3.Naddeo, V. et al. Enhanced ozonation of selected pharmaceutical compounds by sonolysis. Environmental Technology 36, 1876-1883 (2015).

4.Hartmann, J. et al.. Degradation of the drug diclofenac in water by sonolysis in presence of catalysts. Chemosphere 70, 453-461 (2008).

5.Naddeo, V., Meriç, S., Kassinos, D., Belgiorno, V. \& Guida, M. Fate of pharmaceuticals in contaminated urban wastewater effluent under ultrasonic irradiation. Water Research 43, 4019-4027 (2009).

6.Triebskorn, R. et al.. Toxic effects of the non-steroidal anti-inflammatory drug diclofenac. Aquatic Toxicology 68, 151-166 (2004).

7.Ensano, B. M. B. et al.. Applicability of the electrocoagulation process in treating real municipal wastewater containing pharmaceutical active compounds. Journal of Hazardous Materials 361, 367-373 (2019).

8.Ensano, B. et al.. Removal of Pharmaceuticals from Wastewater by Intermittent Electrocoagulation. Water 9,85 (2017).

9.Kamble, S. J., Singh, A. \& Kharat, M. G. A hybrid life cycle assessment based fuzzy multicriteria decision making approach for evaluation and selection of an appropriate municipal wastewater treatment technology. Euro-Mediterranean Journal for Environmental Integration 2, (2017).

10.Tay, K. S., Rahman, N. A. \& Abas, M. R. B. Removal of Selected Endocrine Disrupting Chemicals and Personal Care Products in Surface Waters and Secondary Wastewater by Ozonation. Water Environment Research 83, 684-691 (2011).

11.Kuang, J. et al.. Ozonation of trimethoprim in aqueous solution: Identification of reaction products and their toxicity. Water Research 47, 2863-2872 (2013). 
12.Beltran, F. J., Aguinaco, A., Garcia-Araya, J. F. \& Oropesa, A. Ozone and photocatalytic processes to remove the antibiotic sulfamethoxazole from water. Water Research 42, 3799-3808 (2008).

13.Naddeo, V., Cesaro, A., Mantzavinos, D., Fatta-Kassinos, D. \& Belgiorno, V. Water and wastewater disinfection by ultrasound irradiation - a critical review. Issue 3 16, 561-577 (2014).

14.Torres-Palma, R. A. \& Serna-Galvis, E. A. Sonolysis. in Advanced Oxidation Processes for Waste Water Treatment 177-213 (Elsevier, 2018). doi:10.1016/b978-0-12-810499-6.00007-3.

15.Naddeo, V., Landi, M., Scannapieco, D. \& Belgiorno, V. Sonochemical degradation of twenty-three emerging contaminants in urban wastewater. Desalination and Water Treatment 51, 6601-6608 (2013).

16.Landi, M., Naddeo, V. \& Belgiorno, V. Influence of ultrasound on phenol removal by adsorption on granular activated carbon. Desalination and Water Treatment 23, 181-186 (2010).

17.Prado, M. et al.. Removal of emerging contaminant and fouling control in membrane bioreactors by combined ozonation and sonolysis. International Biodeterioration \& Biodegradation 119 , 577-586 (2017).

18.Naddeo, V., Belgiorno, V., Landi, M., Zarra, T. \& Napoli, R. M. A. Effect of sonolysis on waste activated sludge solubilisation and anaerobic biodegradability. Desalination 249, 762-767 (2009).

19.Dastpak, H., Pasalari, H., Jafari, A. J., Gholami, M. \& Farzadkia, M. Improvement of CoComposting by a combined pretreatment Ozonation/Ultrasonic process in stabilization of raw activated sludge. Scientific Reports 10, (2020).

20.Borea, L. et al.. Wastewater treatment by membrane ultrafiltration enhanced with ultrasound: Effect of membrane flux and ultrasonic frequency. Ultrasonics 83, 42-47 (2018).

21.Naddeo, V., Belgiorno, V., Ricco, D. \& Kassinos, D. Degradation of diclofenac during sonolysis 
ozonation and their simultaneous application. Ultrasonics Sonochemistry 16, 790-794 (2009).

22.Naddeo, V., Ricco, D., Scannapieco, D. \& Belgiorno, V. Degradation of Antibiotics in Wastewater during Sonolysis Ozonation, and Their Simultaneous Application: Operating Conditions Effects and Processes Evaluation. International Journal of Photoenergy 2012, 1-7 (2012).

23.Larcher, S. \& Yargeau, V. The effect of ozone on the biodegradation of 17-ethinylestradiol and sulfamethoxazole by mixed bacterial cultures. Applied Microbiology and Biotechnology 97, 2201-2210 (2012). 
Figure Captions

Figure 1. Rappresentazione esemplificativa e semplicistica riguardante un metodo "ibrido" che combina l'uso di ozono e ultrasuoni per rimuovere i prodotti farmaceutici dalle acque reflue. 


\section{Figures}

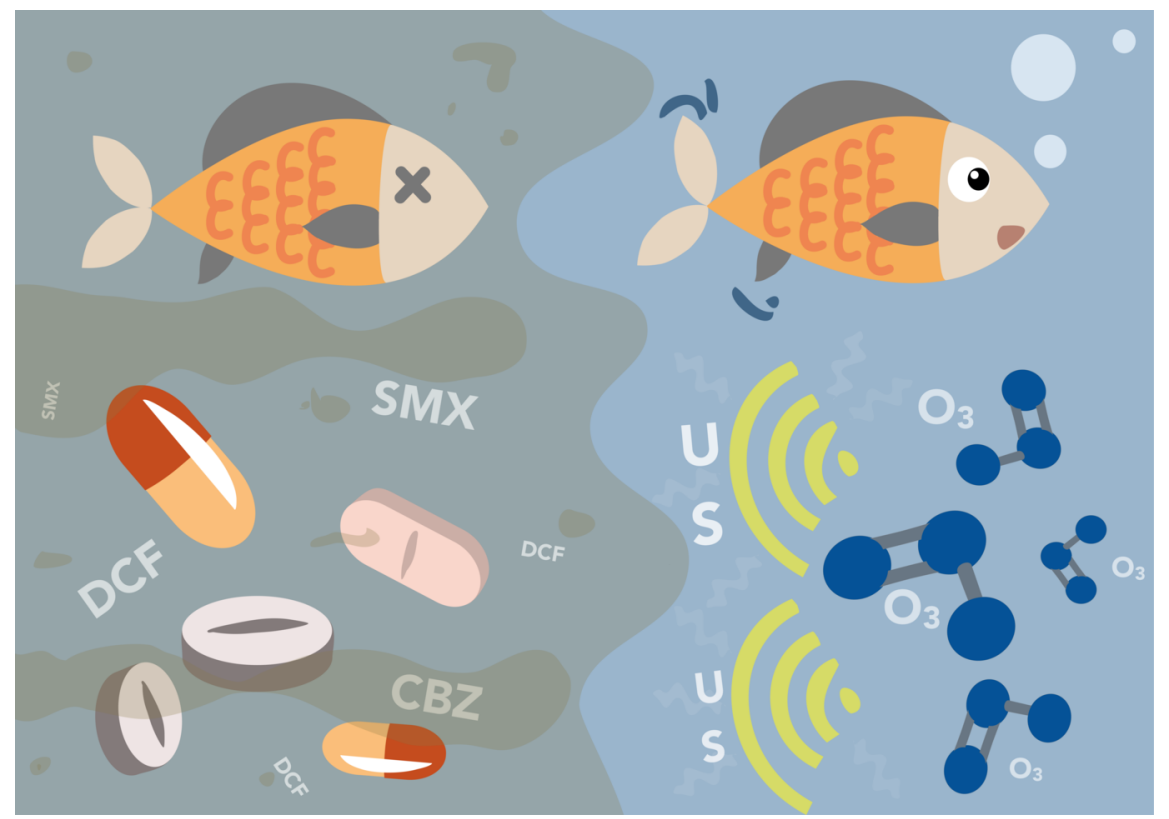

Figure 1: Rappresentazione esemplificativa e semplicistica riguardante un metodo "ibrido" che combina l'uso di ozono e ultrasuoni per rimuovere i prodotti farmaceutici dalle acque reflue. 